\title{
Rat testicular interstitial fluid contains mediators of vasopermeability*
}

\author{
J. M. Tapanainen, T. Paloneva, M. Veijola and H. Rajaniemi \\ Department of Anatomy, University of Oulu, Kajaanintie 52 A, SF-90220 Oulu, Finland
}

\begin{abstract}
Summary. An intradermal injection of testicular interstitial fluid (IF) produced a marked increase in vasopermeability in a dose-dependent manner. Likewise bovine follicular fluid caused a smaller but significant response. The effect of IF was associated with accumulation of polymorphonuclear leucocytes (PMNs) inside the dermal venules and with their adherence to the venular endothelium. A minor but significant response was noticed after injecting anterior chamber fluid, but there was no response after an injection of amniotic fluid or serum intracutaneously. Destroying the Leydig cells with ethane dimethanesulphonate did not change the vasopermeability-increasing effect of IF, but after denaturation of IF proteins the effect was diminished by about $50 \%$. Intravenous administration of hCG did not increase the ability of IF to cause the effect.

These results suggest that rat testicular interstitial fluid contains mediators of vasopermeability, probably specific for the testis and also follicular fluid. The vasopermeability effect of IF does not seem to depend on the collecting time or on Leydig cells and is at least partly mediated by PMNs which are seen in the dermal venules shortly after an injection of IF.
\end{abstract}

Keywords: rat; testis; interstitial fluid; vasopermeability; ovary; follicular fluid

\section{Introduction}

Testicular interstitial fluid (IF) volume appears to be subject to gonadotrophin control. It has been shown that luteinizing hormones ( $\mathrm{LH}$ and $\mathrm{hCG}$ ) increase rat testicular vascular permeability (Sharpe, 1979; Setchell \& Sharpe, 1981) as well as capillary perfusion in the testis (Damber et al., 1981; Setchell \& Sharpe, 1981). The changes in perfusion and permeability drastically increase both the IF volume and the hormone uptake in the testes (Sharpe, 1980, 1981; Veijola et al., 1984; Veijola \& Rajaniemi, 1986). The change in permeability occurs in postcapillary venules (Widmark et al., 1987). Bergh et al. (1986) have reported that the permeability response to hCG is associated with an accumulation of polymorphonuclear leucocytes (PMNs) in the postcapillary venules as well as in the interstitium, and Widmark et al. (1987) have suggested that hCG treatment stimulates secretion of a leucotactic factor(s) in the testes and that the permeability response is PMNmediated. A similar permeability response is obtained when hCG is injected together with testicular interstitial fluid into the testes (Veijola \& Rajaniemi, 1986). We suggested that a direct interaction of hCG with some component(s) present in IF produces a leucotactic factor(s) (Veijola \& Rajaniemi, 1989). Substances in the IF therefore appear to have a significant role in regulation of the testicular interstitial fluid volume. In this study we have examined whether IF contains any endogenous activity that regulates vasopermeability and whether this property is distinctive for testicular IF.

\footnotetext{
*Reprint requests to: Dr Hannu Rajaniemi.
} 


\section{Materials and Methods}

Animals and collection of interstitial fluid. Adult male Sprague-Dawley rats (75-90 days old) weighing approximately $300 \mathrm{~g}$ were used in all experiments. They were anaesthetized with ether before the treatments and before killing. Testicular interstitial fluid (IF) was collected from untreated animals as described by Sharpe (1980). The animal was decapitated, the testes were removed and a careful superficial incision was made in the distal end of the capsule of the testis. Thereafter, the testes were placed into $13 \times 100 \mathrm{~mm}$ conical polystyrene tubes, and IF was allowed to collect over the next $12 \mathrm{~h}$ at $4^{\circ} \mathrm{C}$. The fluid samples were pooled, centrifuged at $100000 \mathrm{~g}$ for $2 \mathrm{~h}$ (Beckman L5-50 B Ultracentrifuge, Beckman Instruments, Inc., Palo Alto, CA, USA) and the supernatants were stored at $-20^{\circ} \mathrm{C}$ until use. Animals $(\mathrm{N}=15)$ were treated with an intravenous injection of $2.5 \mu \mathrm{g} \mathrm{hCG}(10900 \mathrm{i} . \mathrm{u} . / \mathrm{mg}$; Diosynth, Oss, The Netherlands) in $300 \mu \mathrm{l}$ saline $(0.154 \mathrm{M}-\mathrm{NaCl})$. The animals were killed $16 \mathrm{~h}$ later and the IF was collected from the testes for 1,6 or $24 \mathrm{~h}$ as described above. The IF dilutions were made with saline, and a $10 \%$ dilution of IF was used in the experiments, unless otherwise indicated. Some fluid samples were boiled at $100^{\circ} \mathrm{C}$ for $10 \mathrm{~min}$ to denature the IF proteins. The protein deposit was pelleted by centrifuging at $100000 \mathrm{~g}$ for $30 \mathrm{~min}$, whereafter the supernatant was collected. This procedure was repeated once and the final supernatant was stored at $-20^{\circ} \mathrm{C}$ before use in the experiments.

Other ftuids. Samples were collected from two kinds of bovine ovarian follicles. One group consisted of follicles with a diameter of $<5 \mathrm{~mm}$ (small follicles) and the other had a diameter $>10 \mathrm{~mm}$ (large follicles). The ovaries were obtained from a local slaughterhouse. Fluid samples were centrifuged at $100000 \mathrm{~g}$ for $2 \mathrm{~h}$ at $4^{\circ} \mathrm{C}$ and the supernatants were stored at $-20^{\circ} \mathrm{C}$ until use. Amniotic fluid samples were collected from rats at about the 10 th day of pregnancy by amniocentesis. The fluid samples were centrifuged and stored as described above. Anterior chamber fluid was collected by puncture from male rat eyes immediately after killing the animals. The fluid samples were handled as described above.

Depletion of Leydig cells. The Leydig cells were depleted from the testes by treating the rats with ethane dimethanesulphonate (EDS) which is known to destroy Leydig cells of rats (Molenaar et al., 1985; Morris et al., 1986). EDS was synthesized as described by Jackson \& Jackson (1984). The EDS ( $150 \mathrm{mg}$ ) was dissolved in a $5 \mathrm{ml}$ solution of one part DMSO and three parts distilled water, so that the final concentration of EDS was $30 \mathrm{mg} / \mathrm{ml}$. This fluid was given intraperitoneally to rats in a dose of $2.5 \mathrm{ml} / \mathrm{kg}$, so that each rat received $75 \mathrm{mg}$ EDS $/ \mathrm{kg}$. The control groups received, intraperitoneally, $2.5 \mathrm{ml} / \mathrm{kg}$ of a solution of DMSO and water. The animals were killed 3 days later for the experiments and the depletion of Leydig cells was confirmed by histological examination.

Measurement of vasopermeability effect in skin. The method described by Williams (1976) was used with some minor modifications to test the vasopermeability effect. The back skin of adult male rats was depilated 1 day before the experiment by cutting the hair first and then applying the chemical depilator, Immac aerosol. The animals were anaesthetized with ether and they received an intravenous injection of Evans blue $(5 \mathrm{mg} / \mathrm{kg})$ and ${ }^{125} \mathrm{I}$-labelled albumin $\left(12 \times 10^{6}\right.$ c.p.m. $\left./ \mathrm{kg}\right)$, labelled by the chloramine T method (Markkanen et al., 1980), in PBS $(1 \mathrm{ml} / \mathrm{kg})$. The samples to be tested were injected intracutaneously in a volume of $100 \mu \mathrm{l}$ within $5 \mathrm{~min}$. Each rat received 4 pairs of intradermal injections: on one side of the vertebral column the samples to be tested and on the contralateral side the control saline. The animals were killed by decapitation $30 \mathrm{~min}$ after the administration of radiolabelled albumin and dye, they were skinned and the blue patches (Fig. 1) were removed with a steel punch with a diameter of $10 \mathrm{~mm}$ and counted for ${ }^{125} \mathrm{I}$ radioactivity (LKB-Wallac 1280 Ultrogamma, Wallac, Turku, Finland). The permeability change was expressed as a percentage of the radioactivity at the site where the sample was injected to the radioactivity at the contralateral control site.

Statistical analyses. Student's $t$ test for paired data, Mann-Whitney U test and Kruskal-Wallis analysis of variance were used for statistical comparisons.

1

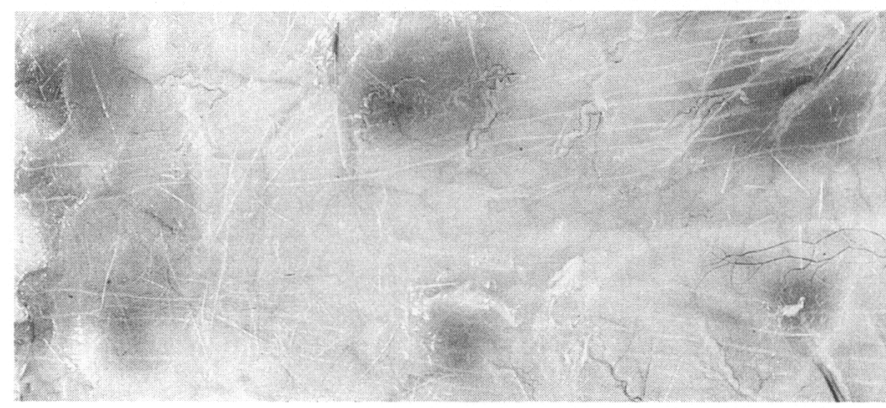

100 IF

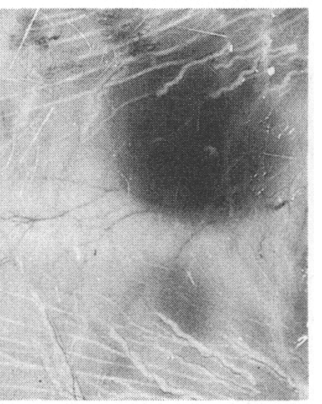

PBS

Fig. 1. Appearance of rat skin within $30 \mathrm{~min}$ after an intravenous injection of Evans blue and ${ }^{125}$ I-labelled albumin and intradermal injections of rat testicular interstitial fluid (IF) in different concentrations (\%, upper row) and phosphate-buffered saline (PBS) as control (lower row). 


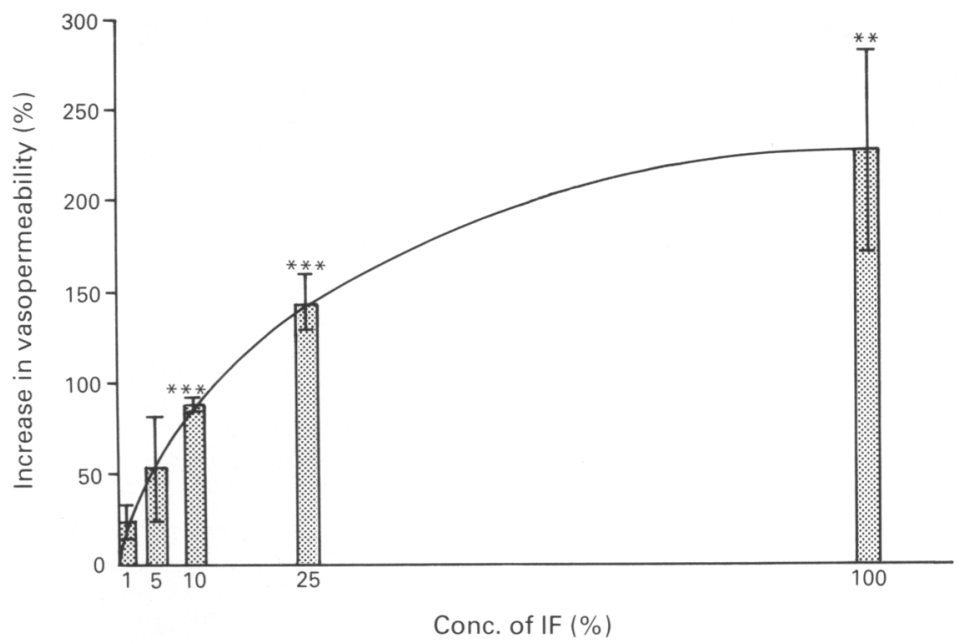

Fig. 2. Effect of different concentrations of IF on vasopermeability in rat skin. A dosedependent increase is noticed. Each column represents a mean \pm s.e.m. The statistical comparison was made by Mann-Whitney $U$ test to the control saline: $1 \%, N=4 ; 5 \%, N=4$; $10 \%, \mathrm{~N}=7 ; 25 \%, \mathrm{~N}=6 ; 100 \%, \mathrm{~N}=3 .{ }^{* *} P<0.01,{ }^{* * *} P<0.001 . P=0.0015$ for all data by Kruskal-Wallis analysis of variance.

\section{Results}

\section{Effect of testicular IF on rat skin vasopermeability}

Testicular IF caused a dose-dependent increase in the vasopermeability of the rat back skin (Fig. 2). The highest increment in permeability $(225 \%)$ was caused by undiluted fluid. No significant difference in the vasopermeability effect of IF samples collected for $1 \mathrm{~h}$ (increase in permeability $\% \pm$ s.e.m.: $114 \cdot 6 \pm 21 \cdot 8, \mathrm{~N}=4), 6 \mathrm{~h}(106 \cdot 0 \pm 24 \cdot 7, \mathrm{~N}=5)$ or $24 \mathrm{~h}(119 \cdot 2 \pm 29 \cdot 9$, $\mathrm{N}=5$ ) was seen. To obtain IF at $1 \mathrm{~h}, \mathrm{hCG}$ treatment was necessary to enhance the formation of IF. The fluid obtained after the treatment was, however, more diluted than the control IF. Therefore, a $50 \%$ dilution was used to obtain a comparable response to the response seen after injecting $10 \%$ dilution of IF from untreated animals. Denaturation of IF proteins seemed to diminish permeability, decreasing the effect by about $50 \%$. The increase in permeability was $239.4 \pm 32 \cdot 4 \%$ (mean \pm s.e.m.; $\mathrm{N}=5$ ) after injecting untreated IF and $112.4 \pm 28 \cdot 7 \%(\mathrm{~N}=5)$ after injecting denatured IF ( $P=0.016$, Mann-Whitney $U$ test $)$.

\section{Effect of other fluids on rat skin vasopermeability}

Bovine follicular fluid caused a significant increase in the rat skin vasopermeability (Fig. 3 ). The response obtained with the fluid collected from the large follicles (diameter $>10 \mathrm{~mm}$ ) was greater but not significantly so, than with the fluid from the small follicles (diameter $<5 \mathrm{~mm}$ ). The increment caused by the fluid from large follicles was about $50 \%$ lower than that caused by undiluted testicular IF. Fluid from the anterior chamber of the eye caused a minor but significant increase in permeability when compared to the saline control, whereas no permeability increasing response was noticed after administration of amniotic fluid or serum. Serum, on the contrary, caused a decrease in the vascular permeability when compared to saline.

\section{Significance of Leydig cells in vasopermeability-increasing effect of IF}

Two kinds of experiments were carried out to elucidate the role of Leydig cells in producing the vasopermeability increasing effect of IF. First, i.v. injection of $5 \mu \mathrm{g}$ hCG in $300 \mu \mathrm{l}$ saline was given to stimulate the Leydig cells. The rats were killed for the collection of IF $6 \mathrm{~h}$ after the 


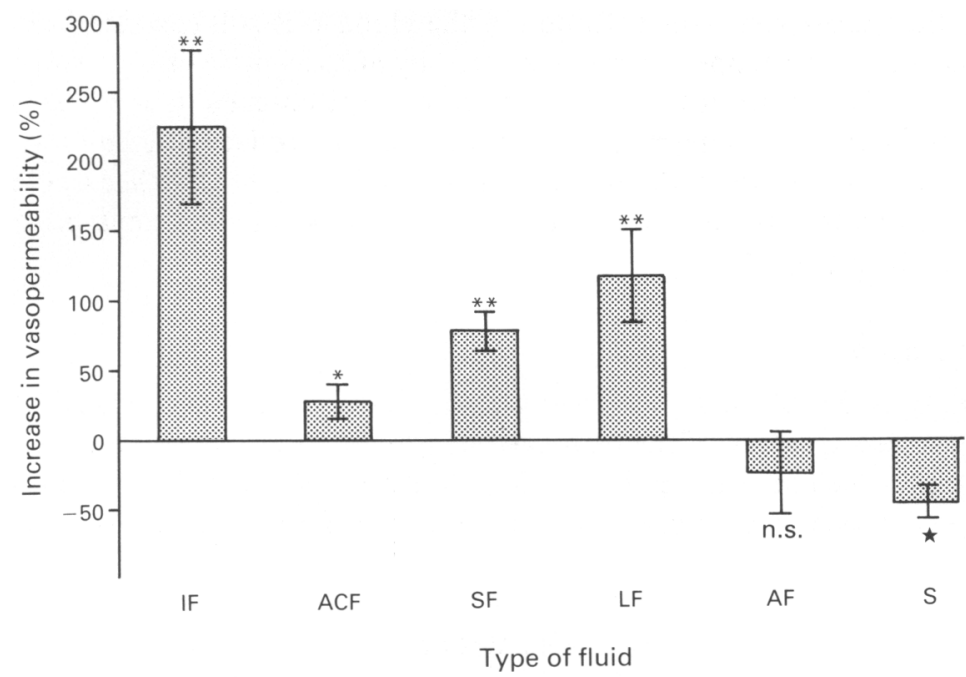

Fig. 3. Effect of different fluids on vasopermeability in rat skin. IF = rat testicular interstitial fluid (undiluted); ACF = rat anterior chamber fluid; $S F=$ bovine follicular fluid, small follicles (diameter $<5 \mathrm{~mm}$ ); $\mathrm{LF}=$ bovine follicular fluid, large follicles (diameter $>10 \mathrm{~mm}$ ); $\mathrm{AF}=$ rat amniotic fluid; $\mathrm{S}=$ rat serum. Each column represents a mean \pm s.e.m. of 3-5 skin patches. The data were compared with the saline control by using Student's $t$ test before converting the values into percentage data. ${ }^{*} P<0.05,{ }^{* *} P<0.01$, n.s. $=$ no significance.

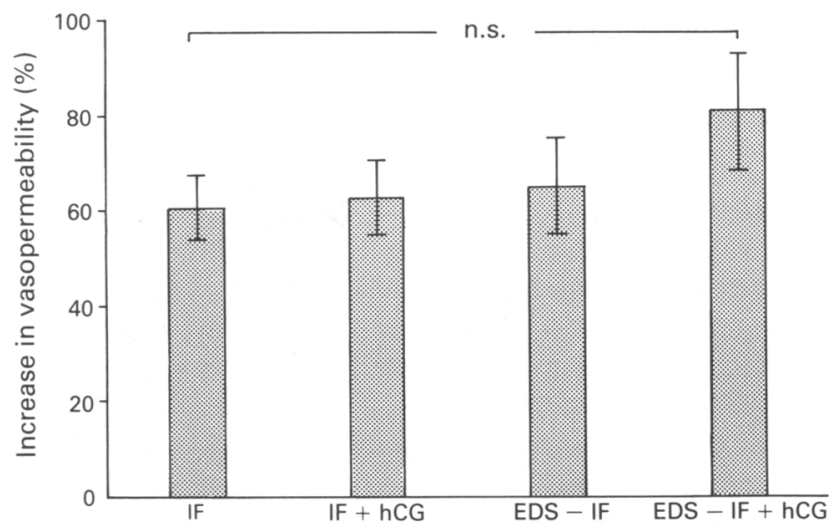

Fig. 4. Effect of treatment of animals with hCG (IF + hCG), EDS (EDS - IF) and with EDS and hCG (EDS - IF + hCG) on the vasopermeability increasing property of IF. Each column represents a mean \pm s.e.m. of 7-8 skin patches. Student's $t$ test and Mann-Whitney U tests were used for statistical comparisons. n.s. = no significance. 
treatment when the testosterone concentration in the blood was still elevated but IF was not yet diluted due to the increased vasopermeability induced by hCG (Veijola $e t$ al., 1984). This IF did not differ from the IF collected from the saline-treated control animals in the vasopermeability response (Fig. 4). Second, the vasopermeability effect of the IF collected from these testes was not decreased when compared to the untreated control animals (Fig. 4). Neither did the treatment of the EDS-treated animals with i.v. hCG produce any significant increment in the permeability increasing activity of IF (Fig. 4).

\section{Histological examination}

Histological specimens of rat skin were made after intradermal injections of 10,50 and $100 \%$ solutions of IF. A clear invasion of neutrophil leucocytes was noticed inside the venules and their adherence to the endothelium was noted (Fig. 5a). Single PMNs were also seen in the interstitium. No such change was seen in sections of skin treated with PBS or in sections of untreated skin (Fig. 5b).

\section{Discussion}

The present results indicate that rat testicular interstitial fluid (IF) is capable of increasing vasopermeability. This property seems to be distinctive not only for testicular interstitial fluid but also for ovarian follicular fluid, as bovine follicular fluid was similarly able to increase vasopermeability of the fluid samples tested. A significant vasopermeability increase was also noticed after injecting anterior chamber fluid but it was minor compared to that obtained with IF or follicular fluid. It is tempting to speculate that the permeability regulating property of interstitial fluid could
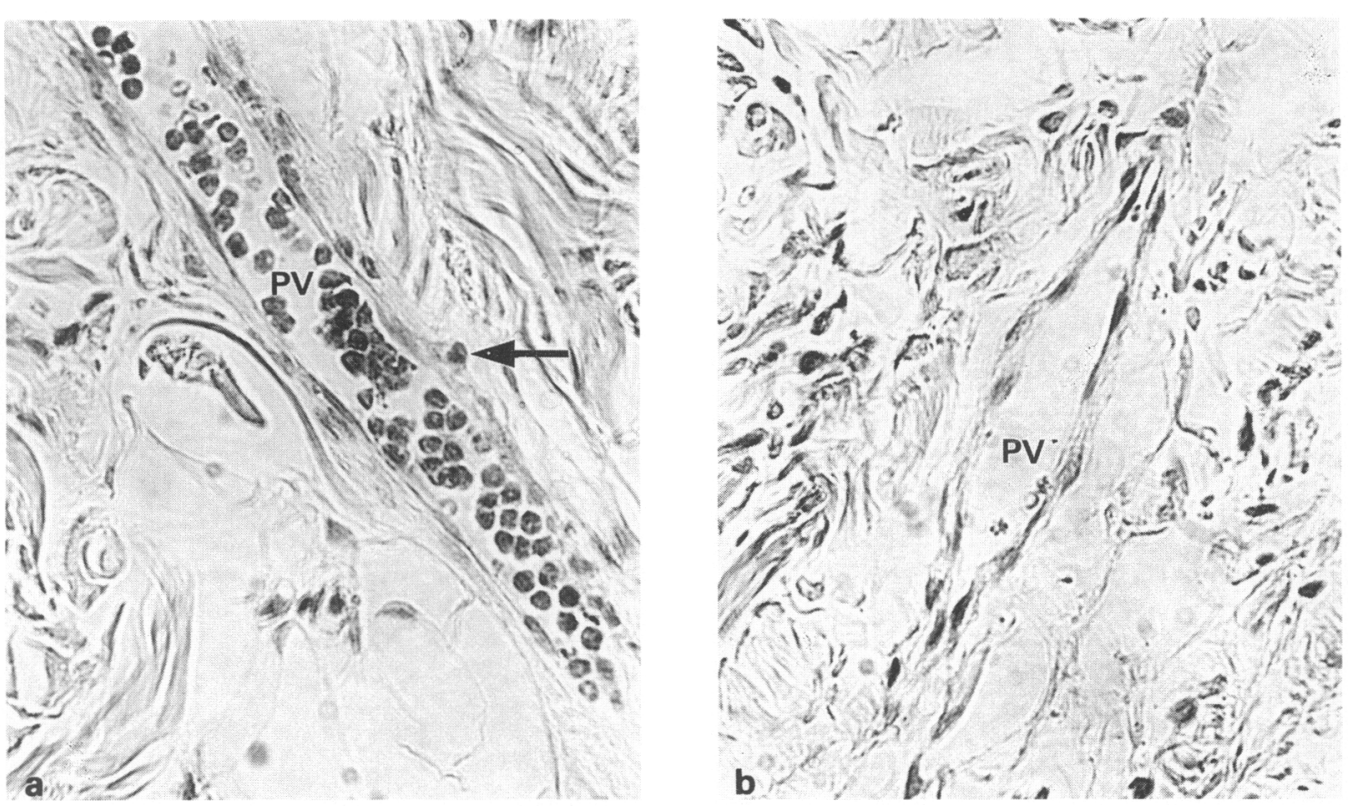

Fig. 5. Photomicrographs of rat skin stained with H\&E $(\times 400)$. (a) Skin treated with $50 \%$ IF. Note that a large number of PMNs is seen in a postcapillary venule (PV), many of them being attached to the endothelium. Only occasional PMNs are seen in the interstitium (arrow). (b) Skin treated with PBS. No accumulation of PMNs is seen in the postcapillary venules (PV) or in the interstitium. 
be unique for most endocrine organs. However, this property is difficult to demonstrate because interstitial fluid from endocrine organs other than testis and ovary is not easily available. As far as the physiological significance of the vasopermeability increasing property is concerned, it seems to provide a subtle way to regulate the interior milieu and thereby the distribution of endocrine and paracrine factors in the organ.

The vasopermeability increasing effect is likely to be mediated by some substance(s) present in testicular IF and in follicular fluid. Some of these substances seem to be proteinaceous as denaturation of IF diminishes the vasopermeability response by $50 \%$. It is also likely that some of them are leucoattractants and that the vasopermeability effect is at least partly mediated by neutrophils because they were seen in large numbers inside the dermal venules as well as attached to the venular endothelium.

Depletion of Leydig cells in the testes did not significantly change the permeability-increasing effect of IF when compared to the control. Nor was any increment in the vasopermeability effect of IF seen after an i.v. injection of hCG. These results suggest that the substances present in IF mediating the permeability response do not originate in the Leydig cells. One can argue that the vasoactive substances are secondarily released to IF, e.g. from macrophages or mast cells during collection of IF. We found, however, that there is no difference in the permeability response between IF samples collected for $1 \mathrm{~h}$ or $24 \mathrm{~h}$.

The present results suggest that rat testicular IF contains vasopermeability increasing substances, some of which seem to be leucoattractants. This property seems to be specific not only for testicular IF but also for follicular fluid.

We thank Ms Aino Kuha, Ms Pirkko Peronius, Ms Eeva Hiltunen, Ms Sirkka Martti and Mr Eero Oja for expert technical and secretarial assistance.

\section{References}

Bergh, A., Widmark, A., Damber, J.-E. \& Cajander, S. (1986) Are leucocytes involved in the human chorionic gonadotropin induced increase in testicular vascular permeability. Endocrinology 119, 586-590.

Damber, J.-E., Selstam, G. \& Wang, J. (1981) Inhibitory effect of estradiol-17\% on hCG-induced increment of testicular blood flow and plasma testosterone concentration in rats. Biol. Reprod. 25, 555-559.

Jackson, C.M. \& Jackson, H. (1984) Comparative protective actions of gonadotrophins and testosterone against the antispermatogenic action of ethane dimethanesulphonate. J. Reprod. Fert. 71, 393-401.

Markkanen, S., Töllikkö, K., Jääskeläinen, K. \& Rajaniemi, H. (1980) Effect of radioiodination on structural integrity, receptor and antibody binding activity and circulatory behavior of human choriongonadotropin. Hormone Res. 12, 32- 45.

Molenaar, R., de Rooij, D.G., Rommerts, F.F.G., Ruevers, P.J. \& van der Molen, H.J. (1985) Specific destruction of Leydig cells in mature rats after in vivo administration of ethane dimethyl sulfonate. Biol. Reprod. 33, 1213-1222.

Morris, I.D., Phillips, D.M. \& Bardin, C.W. (1986) Ethylene dimethylesulfonate destroys Leydig cells in the rat testis. Endocrinology 118, 708-719.

Setchell, B.P. \& Sharpe, R.M. (1981) Effect of injected human chorionic gonadotropin on capillary permeability, extracellular fluid volume and the flow of lymph and blood in the testes of rats. J. Endocr. 91, $245-254$.

Sharpe, R.M. (1979) Gonadotropin-induced accumu- lation of interstitial fluid in the rat testis. $J$. Reprod. Fert. 55, 365-371.

Sharpe, R.M. (1980) Temporal relationship between interstitial fluid accumulation and changes in gonadotropin receptor numbers and steroidogenesis in the rat testis. Biol. Reprod. 22, 851-857.

Sharpe, R.M. (1981) The importance of testicular interstitial fluid accumulation in the transport of injected hCG to the Leydig cell. Int. J. Androl. 4, 64-74.

Veijola, M. \& Rajaniemi, H. (1986) Luteinizing hormones activate a factor(s) in testicular interstitial fluid which increases testicular vascular permeability. Molec. cell. Endocr. 45, 113-118.

Veijola, M. \& Rajaniemi, H. (1989) Interaction of hCG with testicular interstitial fluid produces leucotactic factor(s). Int. J. Androl. 12, 307-317.

Veijola, M., Kellokumpu, S. \& Rajaniemi, H. (1984) The effect of varying doses of hCG on the in vivo uptake by rat testis and serum testosterone response. Hormone Res. 19, 191-199.

Widmark, A., Bergh, A., Damber, J.-E \& Smedgård, G. (1987) Leucocytes mediate the hCG-induced increase in testicular venular permeability. Molec. cell. Endocr. 53, 25-31.

Williams, T.J. (1976) Simultaneous measurement of local plasma exudation and blood flow changes induced by intradermal injection of vasoactive substances using ${ }^{131} \mathrm{I}$-albumin and ${ }^{133} \mathrm{Xe}$. J. Physiol., Lond. 254, 4P-5P, abstr. 